\title{
Architecture as symbolic reverence for nature: case studies: Seed Cathedral - 21st Century and Pigeons' Monastery - 16th Century
}

\author{
E. S. Mashhadi \\ University of Miami School of Architecture, USA
}

\begin{abstract}
Ostensibly unprecedented pieces of architecture, the Seed Cathedral in the Shanghai World Expo and Isfahan's Pigeon Towers in Iran, are two instances where celebrating nature has been formally manifested. In addition to their immediate functions - the former is an exhibition space and the latter a facility which collects birds' droppings as natural fertilizer - they have transcendental intentions: through the formal glorification of the natural, both projects connote the perpetual and timeless interdependencies between the human and nature. By comparing these two buildings, the paper will demonstrate how the same desire has been resurrected into distinct architectural types across time and place.

Beyond their similar ecological intentions, the tectonic logic rooted in the agglomeration of an irreducible unit - the dimension of which is based on the bodily proportions of the organism it accommodates - allows these two artifacts to relate in an intricate way. By focusing on the ecological and sustainable features of the Pigeon Towers, the paper will reiterate architecture's capacity to operate simultaneously in two extreme domains: overlaying the maximum efficiency and the aesthetic perfection. To substantiate the analogy, the paper utilizes Synonymity in Architecture as an operative theory of design to reveal the identical underpinning formation of the two projects. It explains how two ostensibly contrasting artifacts are essentially interrelated.

Keywords: sustainable agriculture, ecological architecture, Pigeon Towers, architecture genesis, design process, typology, Synonymity in Architecture.
\end{abstract}




\section{Introduction}

Although the regard for nature is frequently manifested throughout the history of artifacts, there are few instances where such a respect has reached its apotheosis. Isfahan's Pigeon Towers (Iran), and the Seed Cathedral (China), are two examples in which celebrating nature has been formally expressed (fig. 1).

As an internal presupposition, here I am referring to the Pigeon Towers as a building typology, rather than addressing a particular example. Despite my interest in its typological qualities, and consequently the reluctance to concentrate on a single example par excellence, Hezar Jarib Towers in Isfahan are the most advanced. Scattered across the region, the most sophisticated and matured instances evolved approximately from the 11 th century toward the 17 th century at Iran's central plateau [1]. The towers, as a vernacular type, house thousands of pigeons or doves in order to use their guano as fertilizer.

The Seed Cathedral, the provocative title of the United Kingdom's Pavilion at the Shanghai World Expo, China, 2010, challenges the conventional boundaries through which an object of design can be identified as architecture. With a controversial scheme, a team lead by Heatherwick Studio won the design competition [2]. While the well-precedented approach in designing national pavilions leans toward exhibiting a nation's culture by representing some historic values, Seed Cathedral contravenes this preconception and breaks down the clichés which directly refer to frequently used stereotypes. Abandoning patriotic references, the pavilion privileges creativity over mundane significations. As opposed to symbolizing a historically recognizable and familiar concept, it very consciously ruptures with history and alludes to a more contemporary notion of national identity. It simultaneously exhibits the symbiosis between nature and culture, between architecture and technology.

Setting two radical moments side by side - one contemporary and built with the use of advanced technologies by an individual author, and the other a historically evolved type that defies authorship - the paper transposes them in time to demonstrate how a recurring theme has been resurrected into distinct architectural types spanning natures and cultures; i.e. how the same desire has transcended time, place, and program.

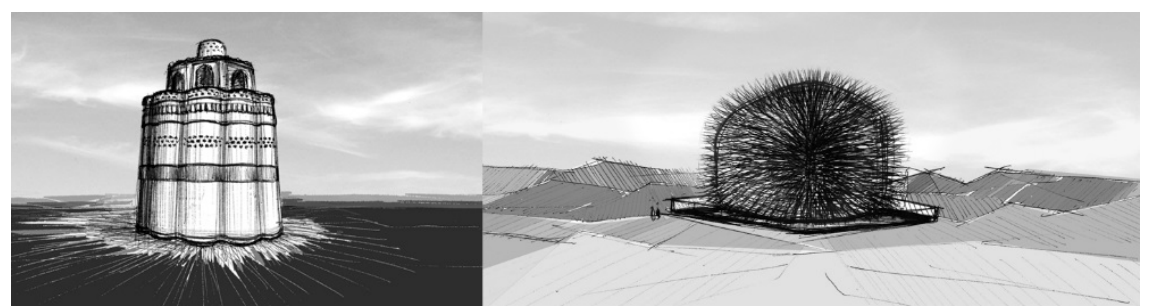

Figure 1: Interior spaces shaped by the repetition. 


\subsection{The Analogy}

The analogy is predicated on both formal and configurational similarities as well as the correspondence of the two projects' underlying intentions. This exemplifies how two ostensibly unlike pieces of architecture are, in fact, synonymous. Their synonymity is not covert, i.e. is not salient on a cursory level, rather it resides in a common genesis. Their corresponding formations, nevertheless, have originated in the repetition of a single cell. The irreducible unit houses organisms: a bird and a set of seeds. In other words, a natural being has shaped the ontology of both projects. To reveal such inherent correlations, this paper incorporates a theory, which I first introduced is Artheo '14 conference, Synonymity in Architecture [3]; which as an analytical tool is capable of explicating the design methodology. Synonymity, expanding the form-content relationship, de-conceals the projects' implicated congruencies. Although from a cursory point of view the function of two buildings might seem unrelated, on a deeper level, their ecological contributions are symmetrical. Having radically different programmatic and functional exigencies (exhibition vs. fertilizer collection), and with essentially dissimilar construction techniques (most advanced vs. rural vernacular), they are both environmentally conscious - the themes are the same, but expressions are distinct.

The next level of analogy addresses the common form-making process. Although from an unsophisticated visual encounter the projects bear subtle similarities: both are symmetrical, free-standing, homogeneous in terms of their material, and can be associated with central configurations; yet, from a more conceptual standpoint, their morphologies have indeed certain rules in common.

\subsection{The apparatus: Synonymity in Architecture}

Despite all the differences in the contents of some buildings, certain aspects of their forms are similar. I have called the similarity in the behaviour of some forms, indifferent to their content, Synonymity in Architecture. Radically different contents pass through a particular process to generate forms. If the utilized process or the syntactical operation is similar, similarity of forms is highly contingent. By expanding the form-content relationship and concentrating on the internal process that gives birth to the forms, Synonymity has both generative and analytical capacities. As opposed to the typological categorization which requires a tangible population of models, the shared qualities of a few instances will be adequate to formulate the processes. This is when Synonymity expands a new horizon.

Since architecture, at least in its historical frame, has been generated to satisfy a need, or accommodate a use, or symbolise a signified, its form is merged into its content. This relationship is forged through the design process. To understand the process, therefore, we need to untangle forms from their contents. In other words, to have access to the underpinning design elements and related mechanisms, it is inevitable to not only investigate the form-content relationship, but to expand the internal stages which collectively generate the form. 


\section{Environmental performance and the evolution of a type}

Although this paper is not meant to be historical research, some facts are critical to the argument. What I refer to as Pigeon Towers evolved through centuries and reached to its performative and aesthetic climax at a felicitous time of national political stability, commercial strength, and artistic dexterity of the Safavid Dynasty, 16th century. These elegant structures have been built to systematize the collection of pigeons' manure to be utilized as fertilizer in agriculture. Beyond this primary use, they were used in the gunpowder industry as well as in the process of tanning leather [4]. Bird's excrement, the guano, is chemically rich manure which can effectively fertilize the soil. The fields were usually planted with melon and cucumber to quench the summer's thirst in Iran's central plateau's hot climate. As opposed to many cultures and regions, in which squabs and pigeons have been considered as a source of food, pigeons have not been bred and housed for such a purpose.

Unlike other types which houses the living creatures and keep them detained, the distinctive quality of the Tower is that the birds are absolutely free. The fact that they are hosted with such a delicacy is not a surprise for a culture which regards birds with symbolic values. An ardent respect for a bird which is the emblem of purity, innocence, and freedom has transcended a merely functional structure to an astonishing type which demonstrates the admirable regard for the nature; and at the same time reveals the phenomenological qualities residing in the relationship of the building with is ground. The intimate relationship between human and nature has been integral to the Eastern culture in general, and to the Persian architecture in particular; where the image of Paradise has been represented into the design of exquisite gardens. A relationship in which Man has not been regarded an autonomous and independent existence dominating the nature and imposing order upon it or to conquer it; but rather to be part of its harmonious being.

\subsection{Design considerations and functional necessities of the Pigeon Towers}

The Towers should have been inaccessible to any external threats. This indispensable commitment poses a challenge to the design: while they are purported to be impervious to impending hazards, they also should provide a convenient entrances for the pigeons [5]. The region's extreme climatic condition is another consideration which renders the slippery solutions futile and nonfunctioning. The Tower's thick walls provide a thermal buffering which moderates the austerity of cold winters and the tyranny of scorching summers.

Although the plan's configurations seems quite complex, there is a simple formula in understanding how the dimensions have been formulated. The exterior wall which is usually tapered and made by the combination of brick, straw clay, lime plaster, and gypsum requires a thickness of 4 to 5 feet [6]. The access/circulation space needs a width of about 10 feet. The inner drum's wall is usually as thick as the peripheral one; and the central space diameter needs to be a minimum of 15 feet. Summing all these ranges together, the overall diameter of 
the turrets ranged from 33 to 78 feet. The height also was very diverse. The proportions was a reliable key for craftsman to estimate the appropriate height which should sustain the vertical and the lateral loads, as well as the pressures resulted from the simultaneous vibration of thousands of birds i.e. to resist the resonance effect.

\section{Beyond performance: two modalities in phenomenology}

The freestanding monumental artifacts (Pigeon Towers) have a dual territorializing capacity. As freestanding landmarks, they serve the birds as an orienting device in the vast unmarked landscape of horizontally stretched fields. Furthermore, the landmarks give a piece of land its identity and its singularity. The repetition of almost identical objects (Towers) conveys the idea that within an approximate radius, the fields belong to the same owner. The slight variations in size, ornamentation, or configuration of each structure are indicative of new boundaries. Beyond their individual capacities in shaping marked places, the distribution of a certain number of edifices shapes a communal notion of place. Whereas the harvest is defining the extension of the land horizontally - which is only recognizable incrementally - these vertical artifacts instantly approximate the limits of the farms.

Several criteria were influential in the rich diversity of the towers: the amount of available labour and financial resources, the quality of craft and mason's skill, the aesthetic taste, etc., were instrumental in differentiating one tower from the other. In addition, each builder was trying to variegate his new structure; thus even the same craftsman altered the new towers. Through this gradual progression of such repetitions over centuries, the Pigeon Towers evolved to an increasingly perfected status.

While by defining the conceptual space around itself, the Pigeon Towers gives the land its sense of place; the Seed Cathedral, on the contrary, defines its own ground. To territorialize an articulated space, distinguished from other adjacent pavilions, it shapes a manifold surface as an irregular plain which empties and thus entails the presence of a unique object. To make a striking figural statement, the design needed to provide enough clarity and visual silence to provoke a more emphatic impression. As a counteraction to the visually cluttered space typical of such exhibitions (overwhelmed by digital screens and multimedia interfaces), the pavilion empties the space for a very tactile and sensuous experience. The monolithic form of the pavilion, deprived of any digital signage and emancipated from motley colors, provides a moment of relief in the visually polluted surrounding spaces. Sitting on a white blanket, the pavilion allows for contemplating the design and its unique experience. The emptied, womb-like space signifies the origin and our dependencies on the Mother Earth. Architecture becomes a medium for advocating nature and the natural.

The fuzzy volume with blurred boundaries liberates the installation from being a conventional piece of architecture. Its tendency to formlessness is an imaginative response subverting the contemporary form-making repertoire. The triangulated platform shapes a topological surface which with its tactile, impervious, and 
variegating characters contrasts the fuzzy, rounded, and translucent qualities of the sculptural piece. As a visual and haptic counterpoint, the crumpled surface stimulates a dialectic between the object and its ground. Together, they set forth a surrealistic image, distorting time and place. Further than such phenomenological qualities, the constructed ground fulfils its pragmatic purpose as well. By accommodating the required functional spaces, the surface, enacts a dual performance in creating an intimate public space above and sheltering spaces below.

\subsection{A metaphor and a paradox}

Like a dandelion, the Seed Cathedral is in a perpetual tension. The bars have been held together, captivated by the center's magnet; yet, there are ready fly away. The objects subvert the nature of the entity they embody: a seed which extends its root deep into the ground has been exhibited in an object which sets forth the sense of levity. A bird, which by definition is all about freedom, is represented in an object which has been nailed down and chained into the ground. The antithetical nature of these objects in regard to stagnation/dynamism constitutes a paradox. Another enigmatic quality has to do with typological confusions. The dandelion-like object affords human's experience of entering a fantasy world; whereas the tower, resembling a monumental religious edifice, privileges the bird, as if they are nuns and monks dwelling in their sanctuary. Such shifts debilitate the typological certainties.

In the case of the Tower, its internal perforated surfaces have embedded in a thick layer of material to sustain its durability and to proof a sealed interior space. Accordingly and inevitably, the perforation is not expressed externally. Further than regular structural function of bearing the vertical load and lateral tensions of a four to five story building, the thick straw-clay wall performs extra tasks: first, it secures the required stability to neutralize the tremendous vibrations generated by simultaneous flapping of thousands of birds [7]. Second, it functions as a thermal buffer in the extreme climatic conditions, moderating the inner temperature. Third, it provides enough poche' spaces for layering the clay blocks which affords the porous spaces of bird's nests. The pavilion, on the contrary, allows its internal formation to extend uninterrupted to shape the external form of the building. This operation demarcates the inside-outside threshold; i.e. the building's form and content are inherently integrated.

The Pavilion possesses only one simple digital feature: the lights embedded inside the rods shed light on seeds and collectively illuminate the space. The resulted effect is the analogue representation of the digital resolution: pixilation of acrylic bars. The spectacle conjures the image of digitally lit screens and is results from the refraction of light along the 7.5 meter acrylic bars dispersed on a compound surface [8]. Through movement, and by the change of distance, the resolution seems altered. The arrangement of 60,000 transparent rods, wrapped around an attenuated form, reacts actively to the natural phenomenon such as sunlight, breeze, and vibrations. This gives the pavilion an atmospheric quality.

Design's animated character reaffirms seeds as the central metaphor, symbolizing the diversity of life. The embryonic nature of seeds has been even 
more dramatically expressed by encapsulating them in transparent blocks, frozen, as if they meant to be saved from extinction. Referring to the expo's main theme, "better city, better life", the pavilion emphasis the indispensable dependency of our existence upon nature and a healthy environment [8]. By doing so, the Seed Cathedral itself becomes a medium. Although it does not possess a remarkable environmental features or sustainable performance, its contribution to the ecological awareness goes beyond the limited physical boundaries of a pavilion. With its implied, yet impressive message, the pavilion turns to an environmental manifesto.

\subsection{Architecture as both the object of desire and a harmonizing machine}

The Pigeon Towers are symptomatic of Heidegger's concept of "fourfold". It strides on the providing ground, signifies the mythical values, serves the community, and unveils the concealed presence of the Divine [9]. It enables the exchange between the avian and human worlds. An architectural type facilitates a series of processes which would otherwise remain disconnected and futile. The astonishing beauty of the Pigeon's Towers instigates a provocative inquiry: why does a specifically functional building (a fertilizer making machine), possess a high degree of aesthetic elegance? It not only has an incredibly sustainable performance in its environmental and ecological functions, but the mythological and ideological values which regard the pigeon as the symbol of freedom, innocence, and purity, have been conducive to the evolution of a symbolic form; the exquisite beauty of which supersedes its performative necessities. Hence, the tower creates a space which conjures the image of, and the sacred whisper of a cathedral.

The desire to express beauty in any cultural production in the most deserved manner has been corroborated with a rich history of architectural practice in a wide spectrum of types. The Pigeon Towers brings history, materiality, mythology, morality, and nature all together, captured in a single time-space frame.

\subsection{From performance to ontology}

How a vernacular/rural type has achieved a degree of self-consciousness to hypothesis the aesthetic imagination of a bird. Its remarkable achievement can even be better realized if we compare it with another solution: a scarecrow! Only occasionally successful in repelling the birds, the scarecrow operates through a primitive, literal personification of a human; whereas the Tower's approach is mature and far more advanced. The ingenious application of color, texture, and scale has rendered the Towers a magnificent asset among other fascinating Iran's architectural typologies. As an inviting monumental icon to pigeons, the tower generates the image of an impervious fort to dissuade and repel invaders and predators [10].

If a single nest has been the birds' instinct solution for an individual place of dwelling, a single nest, nevertheless, other types of collective habitations are evident in the case of other species. Beehives are a pertinent example of such a communal dwelling. Interestingly, both the Pigeon Towers and the British 
Pavilion are analogous to the formal structure of a beehive. An inquiry is stimulating: how is the form of a hive related to the shape of these building? The application of a same technique generates two essentially synonymous interiors. The revolution of a honeycomb surface around a center composes a perforated cylindrical drum which simulates the interior fabric of the Tower. Wrapping the interior of a chamfered cube with the same honeycomb texture, and simultaneously extruding each hexagon outward, the enclosed space of the Pavilion will reproduced (fig. 2).

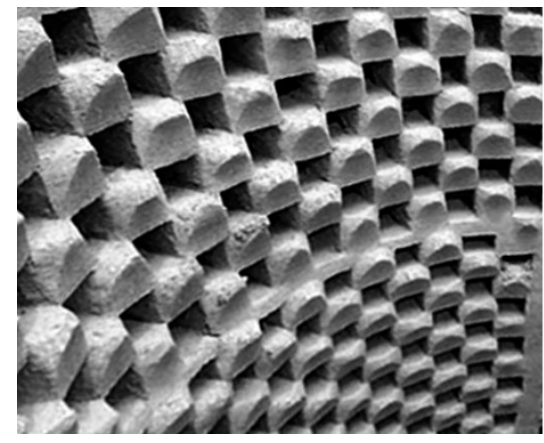

(a)

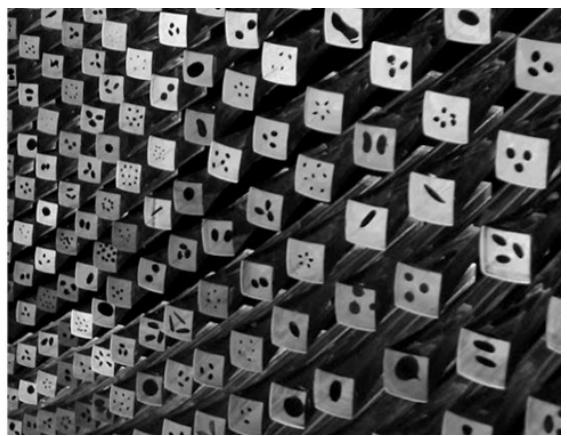

(b)

Figure 2: Interior spaces (a) Pigeon Tower; (b) Seed Cathedral.

\section{Transposition of two traditions and the evolution of a type}

How do we translate the relatively small scale, derived from the dimension of a bird, to a magnificent building with a scale comparable to a windmill? It is wonderful how a type has evolved through combination of completely different forms, from radically different scales. Many of the historical free-standing types with central plans, such as many cylindrical mausoleums (Kharraqan Tower near Qazvin), or many tombs and turret towers (Tuğrul Tower in Ray, Mil-i Radkan in Radkan), could be regarded as formal precedents for the Pigeon Towers. Centuries of knowledge and experience in clay construction and brick tectonics have substantiated the emergence of the Pigeon Towers as a hybrid type. The appropriations of other typologies, as an external inspiration, and the brick screening, as an internal genesis, have contributed to the emergence of such an astonishing phenomenon. The former operation which organizes the project by the imposition of a totalizing concept, borrowed from external but relevant typologies, is identified as top-bottom process; and the later which shapes the space through an internal and intrinsic operation, with bottom-top design.

\subsection{Simultaneous coexistence of two approaches in design}

The functional and performative necessities of the Towers have mediated the topdown impositions. For instance, the central cylindrical shaft, or the central drum, 
usually extends higher than the external tube. The core continues higher to create a central anchor which simultaneously decreases the structural spanning, and acts as an inward buttress. It also facilitates the natural ventilation and operates as a cooling tower, a phenomenon known as the Chimney Effect or Stack Effect. Another performance-driven transformation is evident in a decorative frieze-like ribbon somewhere close to the parapet - or below the punctured holes that the birds enter. Further than its aesthetic qualities, the slippery frieze is a trap for snakes that prevents them from climbing the tower all the way toward the fenestrations [12].

\subsection{Repetition as genesis: actualizing an inherent tendency}

Their shared bottom-top design technique is based on repetition and agglomeration. The tectonics is based on the accumulation of pieces, the scale of which is originated in the dimensions of natural entities. In other words, to shape the space, they agglomerate an irreducible unit - a bodily physical dimension. One establishes its scale based on the pigeon's proportions, and the other articulates seeds in distinguishable parcels so that their physical characteristics (shape, color, and details) become legible. It then elevates the aggregation of a small piece to a monumental scale.

Along with New Materialist discourses which consider matter to have morphogenetic capacities [13], brick has exhibited a vibrant range of such spontaneously inclinations. As a singular object it has an innate tendency to connect and unite to shape a coherent whole. Repetition, therefore, is an integral quality of the brick's tectonics which has not only led to innumerable patterns in variety of configurations (linear, curvilinear, manifold, striated, stepped, screened, and so on), but also has developed historically through certain construction techniques. Following this tradition, masons harnessed the brick's tectonic performance to allow its latent qualities to be expressed, unfolding brick's morphogenetic potentials; i.e. to deliver what is already pregnant in that material.

Here, the repetition actualizes itself into the shape of cylinder through bending around a center. The polarity of an imaginative surface transforms a straight brick screen to a drum-like form. Adding an extra attractor such as a rhythmic differentiation, issues a far more complex space [14]. Introducing new constraints to this generative system - such as culling out the empty units - delivers a porous space. By adding or leaving one unit empty every other space, a dashed pattern emerges. To continue stacking this rhythmical solid-void pattern, the wall is required to shift vertically. Over adding a phase of displacement to the next row, a porous screen surface appears. In this way, a very simple algorithm, based on the space of possibility of a material, formulates the tower's geometry. To create an enclosed space out of the resulting perforated clay surface, the building requires a central plan. As opposed to a square parti that generates corners which would not be accessible, the circular plan provides the maximum accessible surface area.

Now if the remained inner area radically exceeds a convenient working area, the next cylinder can be inserted inside; and if wide enough it can be hollowed out. One layer of the punctured surface, therefore, expands to three (fig. 3). This increases the number of doves and of course the produced fertilizer. 
Another ingenious move has been the use of interior protrusions to buttress the tall structure without building extremely thick walls to expand the available space. The entire surface of these buttresses has been covered by hundreds of nests. Even more impressively, in some variations the perfected cylinder transforms to a series of undulated curves by introducing sub-centers. This asterisk patterns compartmentalizes the inner space in more intricate and efficient way, while allows the tower's facade to be more aesthetically appealing.
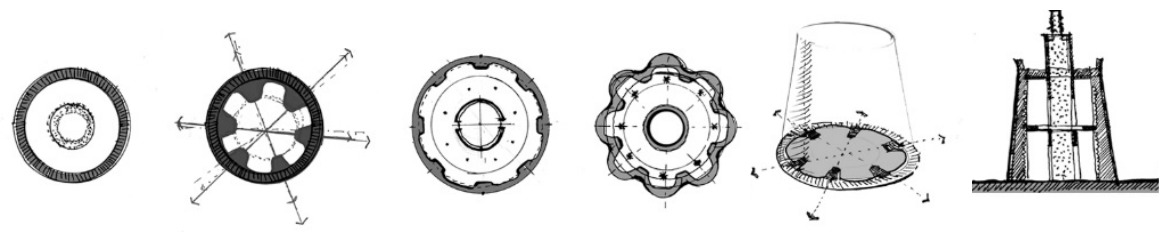

Figure 3: Evolution of Pigeon Towers.

\subsection{Texture and the genesis}

Texture, is not only the visually prominent aspect of both projects, but more importantly, it has been fundamental to their inception as well (fig. 4). In the case of Pavilion texture is simultaneously expressed as both the interior and exterior, while for Tower, the necessity of providing an absolutely enclosed space could not allow the texture to be expressed externally. In the Pavilion, the repetition has an inbuilt, differentiation: each cubic bar embodies a different set of seeds to exhibit the diversity of 250,000 existing kinds. Where the texture revolves around a center, the 60,000 rods are configured into a manifold surface. Despite its central and symmetrical organization, the manifold generates an astonishing visual effect. Similar spatial quality is evident in Tower as well. The optimization of spaces spontaneously entails doubled, or tripled layering, which gets even more complicated as it embraces sectional divergence to provide farmer's access to the mezzanine level.
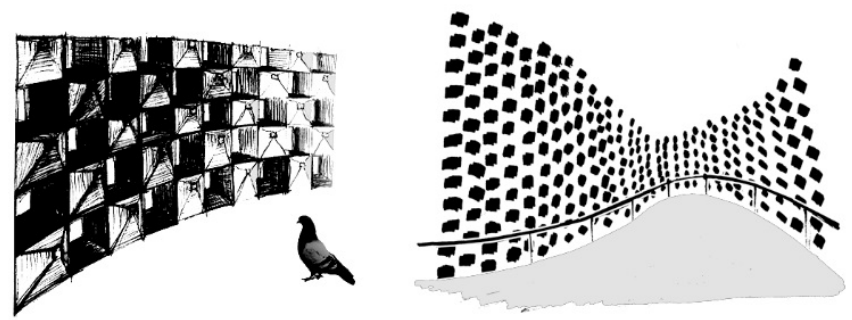

Figure 4: Repetition, texture and genesis.

Based on their interchangeable process, the same generic algorithm can create either of projects. By the change of certain parameters - the way that computer coding in parametricism operates - each interior is transformable to the other [15]. 
In the evolution of Pigeon Towers, likewise, almost any of the towers maintained its singularity when sharing a collective identity. Interchangeability of the Tower and Pavilion goes beyond a typological formulation. However, Synonymity as an operative theory is capable of explaining conditions which typology is not extended to. As a complementary theory it demonstrates the two projects identical form-making process.

\section{Conclusion}

Except for moments of negligence, architecture has always used any opportunity to express its regard for Nature and the natural. Every single window tries to invite life in, and (even though conceptually) to unite with the infinity of our world. The fact that the same desire has frequently revived into different forms, different places, and different moments, reaffirms that architecture's vocation in glorifying Nature, is eternal and universal.

One houses birds as free and animated; the other arranges assorted sets of seeds as captured and petrified. Although the former has an eminent functional purpose, and the latter has an ambition to represent a new concept of design into a fascinating form, they both have one overriding desire in common. Both projects highlight the perpetual and timeless symbiosis between the human and nature - an intimate felicitous correlation which has been occasionally neglected and put aside by post-industrial societies.

The multifaceted design of the Pigeon Towers is a propitious compromise: from the technical point of view, it is the paragon for sustainable design, encompassing concepts such as maximum efficiency, zero emission, and sustainable agriculture. From a semiotic perspective, its significations extend beyond expectations. Its aesthetics conforms to both human's and bird's visual demands. It elevates the dovecote typology to a higher level. Some other instances of this typology typically resemble crude industrial facilities. Their design has been concerned with a provincial approach to their practicality. Hezar Jarib Towers, on the contrary, exhibit extra glamour. The Towers provoke wonder not only based on their sustainable performance but more dramatically in their exquisite aesthetic perfection; which cannot be justified without understanding the affective narratives residing in their formation. The emotional, symbolic, and mythical associations have transcended a merely functional structure to an emblem of reverence for Nature.

Based on an interchangeable design technique, Pigeon Towers and UK's Pavilion in all their differences - time, place, culture, technology, materials, and function - are fundamentally interrelated. What authorizes the synonymity of these two supposedly contrasting buildings is not only the equivalence of their underpinning environmental intentions, but, on a more physical and tangible level, their identical genesis.

\section{Acknowledgements}

I would like to thank my director, Prof. J-F. Lejeune, the Director of Graduate Studies at the University of Miami School of Architecture, for opening new 
horizons which would have otherwise have remained hidden. Prof. Katherine. J. Wheeler's methodological and theoretical contributions were critically invaluable and exceeded the scope of this research. Prof. Denis Hector's comments and inspirations regarding the tectonic logic of the two projects enriched this research considerably.

All illustrations belong to or drawn by the author unless otherwise noted.

\section{References}

[1] Damirchi, A. Pigeons and the pigeon towers of Isfahan, Journal of Art and Mankind (115), pp. 34-37, 2004.

[2] Heatherwick Studio website, UK Pavilion, Online, http://www.heatherwick.com/uk-pavilion

[3] Sheikholharam M. E. Synonymity in Architecture. The Similarity of Forms and a Theory of Design. ARTHEO'14 / Philosophy, Aesthetic and Theory of Art Conference, Turkey, pp. 2-5, 2014.

[4] Beazley E., Pigeon towers and ice-houses on the Iranian plateau, Technology, Tradition and Survival: Aspects of Material Culture in the Middle East and Central Asia / eds. R Tapper, K. McLachlan. Frank Cass, London, p. 75, 2004.

[5] Pratt, J. G. An Investigation of Homing ability in Pigeons without previous homing experience, Journal of Experimental Biology (32) pp. 70-83, 1954.

[6] Amirkhani, A., Okhovat, H., \& Zamani, E. Ancient pigeon houses: Remarkable example of the Asian culture crystallized in the architecture of Iran and Central Anatolia. Asian Culture and History, 2(2), pp. 45-57, 2010.

[7] Mattewes, G.V.T., the Experimental investigation of navigation in homing pigeons, University of Cambridge. p. 61, 1951.

[8] Heatherwick Studio Website, http://www.heatherwick.com/uk-pavilion/

[9] Critchley, P., Martin Heidegger: Ontology and Ecology In: P. Critchley, The City of Reason, (7), The Ecological Concept of the City, p.3, 2004.

[10] Bourgeois J., Pelos C., Spectacular Vernacular: A New Appreciation of Traditional Desert Architecture, Peregino Smith Books, New York. pp. 9195, 1983.

[11] Ferrier, R.W. The Arts of Persia. Yale Press, New Haven. pp. 54-58, 1989.

[12] Delanda. M., Deleuze and the Use of the Genetic Algorithm in Architecture, Lecture. Art and Technology Lecture Series, Columbia University, 2004.

[13] Carpo, M. Digital Darwinism: Mass Collaboration, Form-Finding, and the Dissolution of Authorship. Digital Style Fall, Log 26, 2012. http://www.anycorp.com/anycorp/article/125 